\title{
Fast and effective dimensioning algorithm for end-to- end optical burst switching networks with ON-OFF traffic model
}

\author{
Reinaldo Vallejos ${ }^{1}$, Alejandra Zapata ${ }^{1}$, Marco Aravena ${ }^{2,1}$ \\ 1 Telematics Group, Electronic Engineering Department, Universidad Técnica Federico \\ Santa María, Chile \\ reinaldo.vallejos@usm.cl, alejandra.zapata@usm.cl \\ 2 Computer Science Department, Universidad de Valparaíso, Chile \\ marco.aravena@uv.cl
}

\begin{abstract}
A novel algorithm for fast dimensioning of end-to-end optical burst switching networks is proposed. The proposed method determines the number of wavelengths for each network link according to the traffic load, the routing algorithm and the required blocking probability per connection. The burst input traffic is modeled by an ON-OFF alternating renewal process, which is more realistic for OBS networks than the typically used Poisson model. Compared to the two most typically used dimensioning approaches, the proposed method results in significant lower wavelength requirements whilst achieving the same target blocking probability. Additionally, the proposed method takes less than one second to dimension the network links which makes it several orders of magnitude faster than the conventional simulation approach.
\end{abstract}

Keywords: WDM networks, OBS networks, ON-OFF traffic, network dimensioning, blocking probability.

\section{Introduction}

The high bandwidth requirement imposed by the ever increasing amount of Internet traffic can only be met by using Wavelength Division Multiplexing (WDM) networks, currently allowing bandwidths of the order of 1-10 Tb/s per fibre and already used in transport networks as high-speed transmission channels throughout the world.

At the moment, however, the potential of WDM channels could not be fully utilised by already successful networking approaches as packet-switching because optical buffering and processing is not mature enough [1,2]. Alternatively, these tasks could be carried out electronically, but electronic operation cannot match the high speed of optical transmission.

As a result, a new switching paradigm -feasible in the short term- has been proposed for WDM networks: optical burst switching (OBS) [3-6], which integrates the fast transmission speed of optical channels and the electronic capability of electronic nodes. OBS networks aim to decrease the electronic speed requirements imposed by the optical transmission rate and the packet-by-packet operation- by 
aggregating packets at the edge of the network. The electronic aggregation of packets is into a container, so called burst, and intermediate optical network resources are allocated burst-by-burst. Therefore, the demanding switching and processing speed requirements (which are served electronically) are relaxed and data transmission is carried out at maximum speed. The overhead incurred by operating in this way is the time spent in the reservation of optical resources whenever a burst must be transmitted (and the time spent in the corresponding resource release).

OBS networks can operate in two ways: in a hop-by-hop or in an end-to-end basis. In the first case, the burst is optically transmitted through the network just after a control packet has configured switches on a hop-by-hop basis [3,4]. However, bursts can be dropped at any point along the path to destination due to wavelength contention. In the second case, the burst is transmitted only after all the resources from source to destination have been reserved [5,6]. In [5] this type of network was termed WR-OBS (wavelength-routed optical burst switching) network. This paper focuses on end-to-end OBS networks as it has been shown to significantly reduce the burst loss rate with respect to a hop-by-hop OBS network of equivalent complexity [7].

Given that the number of wavelengths impacts significantly the network cost, the efficient dimensioning of every link in an end-to-end OBS network is of paramount importance. Usually, the dimensioning of OBS networks has been done assuming that all the network links have the same number of wavelengths, see for example [8-10]. However, this approach leads to over-dimensioned networks with under-utilised links. An alternative approach for networks using fixed routing has been to allocate as many wavelengths to a link as connections using such link (this is equivalent to the capacity required in an equivalent static network), see for example [11,12]. However, this method does not take advantage of the statistical multiplexing of connections, especially at low traffic loads. Finally, in the context of hop-by-hop OBS networks, the application of the Erlang-B (under the assumption of a Poisson process arrival of bursts) formula to quantify the wavelength requirements of the network links as a function of the link blocking has been used [12, 13]. However, a Poisson process (for which a well developed theory exists) is not representative of the real burst traffic offered to the optical core because one main property of the Poisson process is that its arrival rate does not change on time. This makes the Poisson model unsuitable for applications where this property does not hold. In an OBS network the total number of bursts that might be simultaneously sharing a given link is small (no more than a few tens in networks of practical interest) and the arrival rate at any instant to any link depends on the number of connections (from the total using such link) that are inactive. Thus, the arrival rate changes significantly when a single connection switches its state, which makes the Poisson model unsuitable for the burst traffic of OBS networks. Instead, the burst arrival process naturally follows an ON-OFF process: a source is in OFF mode during burst aggregation and in ON mode during burst transmission [14-16].

In this paper, the dimensioning of end-to-end OBS networks is carried out by means of a new algorithm which considers the ON-OFF nature of burst traffic, the required blocking probability of connections and the routing algorithm. Results show that the proposed method achieves a much lower wavelength requirement than conventional approaches whilst guaranteeing that the blocking probability per 
connection does not exceed a specified threshold. Additionally, the proposed method is much faster than any conventional approach exploiting the statistical multiplexing gain (typically based on the sequential execution of simulation experiments). These features make the proposed method a natural choice for implementation in future dynamic OBS networks.

The remainder of this paper is as follows: section 2 presents the network and traffic model and introduces the notation used throughout the paper; section 3 presents the proposed method whilst numerical results are presented in section 4 . Section 5 summarizes the paper.

\section{Network and traffic model}

\subsection{Network architecture}

The architecture studied corresponds to an optical OBS network with end-to-end resource reservation and full wavelength conversion capability, as it has been shown that wavelength conversion improves the blocking performance of dynamic WDM networks and it is key for dynamic networks to achieve wavelength savings with respect to the static approach, see for example $[17,18]$.

In such an optical network architecture each node consists of an optical switch locally connected to an electronic edge router where incoming packets are classified according to destination (see upper section of Fig. 1). Every edge router is equipped with one buffer per destination, where burst aggregation is carried out. During the burst aggregation process a resource request is generated once a pre-defined condition is met (e.g. latency or burst size), and such request is sent through the network to reserve resources for the burst (see lower section of Fig. 1). In this paper requests are assumed to be processed according to a fixed routing algorithm [19]. If such algorithm finds an available route, an acknowledgement is sent to the corresponding

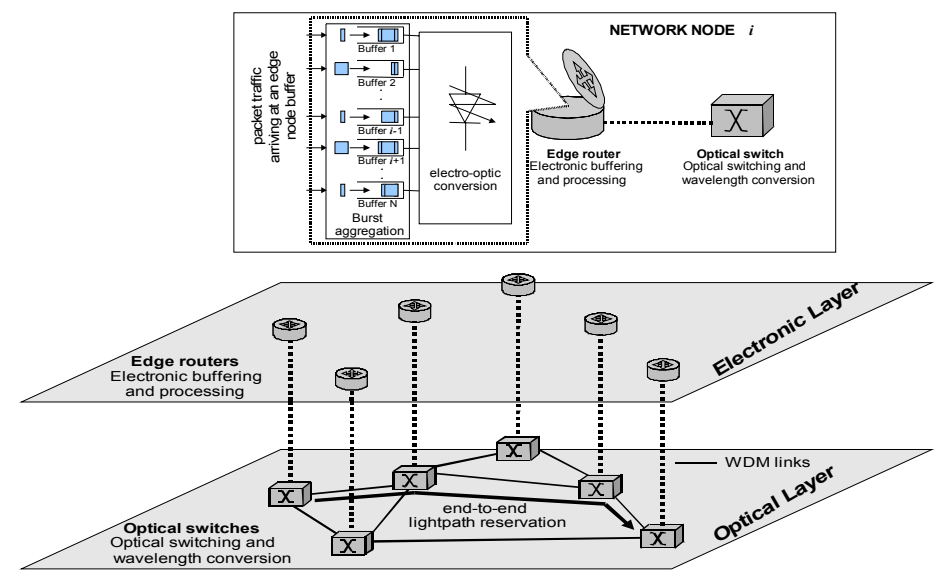

Fig. 1. Schematic of an end-to-end OBS network 
source node, the network is configured to establish the end-to-end route and the burst is released into the optical core. Otherwise, the request is dropped with a NACK (Negative Acknowledgement) message sent to the source node which blocks the burst from entering the network.

\subsection{Network Model}

The network is represented by a directed graph $G=(\mathcal{N}, \mathcal{L})$ where $\mathcal{N}$ is the set of network nodes and $\mathcal{L}$ the set of uni-directional links (adjacent nodes are assumed connected by two fibers, one per direction). $N$ and $L$ represent the cardinality of the set $\mathcal{N}$ and $\mathcal{L}$, respectively. The capacity of the link $l \in \mathcal{L}$, in number of wavelengths, is denoted by $W_{l}$. The aim of this paper is to determine the minimum value for $\sum_{\forall l \in \mathcal{L}} W_{l}$ such that the value for the blocking probability of each connection is guaranteed not to exceed a given threshold.

\subsection{Dynamic traffic model}

The burst aggregation process transforms the original packet traffic into a burst traffic which can be described by a source which switches its level of activity between two states: ON (burst transmission) and OFF (time between the end of the transmission of a burst and the beginning of the following one). During the ON period, the source transmits at the maximum bit rate (corresponding to the transmission rate of one wavelength). During the OFF period, the source refrains from transmitting data. This behaviour has been modelled by an ON-OFF alternating renewal process, for example in [14-16], and it is also used in this paper to characterise the burst traffic generated at the edge nodes.

In the network there are $N(N-1)$ different connections (source-destination pairs). In this paper, each connection is mapped to a number -denoted by $c$ - between 1 and $N(N-1)$. Each connection $c$ is associated to a route, $r_{c}$, determined by a fixed routing algorithm and established only during the $\mathrm{ON}$ period of connection $c$.

According to the usual characterization of an ON-OFF traffic source [20], the demand of connection $c$ is defined by two parameters: mean OFF period duration (denoted by $t_{O F F}$ ) and mean $\mathrm{ON}$ period duration (denoted by $t_{O N}$ ), equal for all connections (homogeneous traffic). The mean ON period includes burst transmission as well as the time required to reserve/release resources for the burst. The latter depends on the reservation mechanism used, which is out of the scope of this paper. According to the alternating renewal process theory [21], the traffic load offered by one connection, $\rho$, is given by $t_{O N} /\left(t_{O N}+t_{O F F}\right)$. The traffic load can be also though as the stationary state probability that a given connection is in $\mathrm{ON}$ state. 


\section{Dimensioning algorithm}

The dimensioning algorithm proposed in this paper aims to guarantee that the blocking $B_{c}$ for each connection $c$ does not exceed a given threshold $B$ (typically agreed in the Service Level Agreement between carriers and customers).

By assuming link blocking independence, the blocking probability of connection $c$ is given by the following expression:

$$
B_{c}=1-\prod_{\forall l \in r_{c}}\left(1-B_{l}\right) ; \quad 1 \leq c \leq N(N-1)
$$

where $l$ denotes a link in the route $r_{c}$ and $B_{l}$ is the blocking probability of link $l$.

Let $H_{c}$ be the length (in number of hops) of the route $r_{c}$. There are many factors (of the form $\left(1-B_{l}\right), \forall l \in \mathcal{L}$ ) satisfying Eq. (1), including the one where all the $H_{c}$ factors have the same value. To facilitate analytical treatment of Eq. (1) without modifying the target of the dimensioning process (to guarantee that the blocking $B_{c}, 1 \leq c \leq$ $N(N-1)$, does not exceed a given value $B$ ) the same value of $B_{l}$ for all links in the route is used. This leads to the following expression:

$$
1-\left(1-B_{l}\right)^{H_{c}} \leq B ; \quad 1 \leq c \leq N(N-1)
$$

where $H_{c}$ is the length (in number of hops) of the route $r_{c}$.

Because the different connections using a given link $l$ might have different route lengths, to guarantee that all connections (even the longest) achieve a blocking probability lower than $B$, the value of $H_{c}$ corresponding to the longest route using link $l$ is used in Eq. (2). Thus, the link $l$ must be dimensioned so the following condition is met:

$$
B_{l}=1-\sqrt[\hat{H}_{l}]{(1-B)} ; \quad \forall l \in \mathcal{L}
$$

where $\hat{H}_{l}$ corresponds to the length (in number of hops) of the longest route using link $l$.

\subsection{Link blocking evaluation}

Let $W_{l}$ be the number of wavelengths of link $l$. Then, the blocking probability of link $l$ is given by the following expression $[22,23]$ :

$$
B_{l}=\lambda_{l}\left(W_{l}\right) \cdot P_{l}\left(W_{l}\right) / \lambda_{l} ; \quad \forall l \in \mathcal{L}
$$

where $\lambda_{l}(w), 0 \leq w \leq W_{l}$, is the mean arrival rate of connection requests to link $l$ when $w$ wavelengths are in use, $\lambda_{l}$ the mean arrival rate of connection requests to link $l$ and $P_{l}(w), 0 \leq w \leq W_{l}$, the probability of $w$ wavelengths being used in link $l$. That is, the link blocking corresponds to the ratio between the mean number of requests arrived (per 
time unit) when the link is fully used $\left(\lambda_{l}\left(W_{l}\right) \cdot P_{l}\left(W_{l}\right)\right)$ and the mean number of requests arrived to the link $\left(\lambda_{l}\right)$. Next, expressions for: a) $\lambda_{l}(w)$, b) $\lambda_{l}$ and c) $P_{l}(w)$ in Eq. (4) are given.

a) Let $T_{l}$ be the total number of connections whose routes use link $l$ (in general, $T_{l}>W_{l}$, due to the statistical multiplexing gain) then:

$$
\lambda_{l}(w)=\left(T_{l}-w\right) \cdot \lambda ; \quad 0 \leq w \leq W_{l}, \forall l \in \mathcal{L}
$$

where $\lambda=1 / t_{\text {OFF }}$ corresponds to the mean burst arrival rate of one connection (when it switches to the OFF state).

b) From the mean value definition:

$$
\lambda_{l}=\sum_{w=0}^{W_{l}} \lambda_{l}(w) \cdot P_{l}(w) ; \quad \forall l \in \mathcal{L}
$$

c) Finally, $P_{l}(w), 0 \leq w \leq W_{l}$, can be approximated from:

$$
P_{l}(w)=P^{*}\left(w / T_{l}\right)\left[\sum_{w=0}^{W_{l}} P^{*}\left(w / T_{l}\right)\right]^{-1} ; 0 \leq w \leq W_{l}, \forall l \in \mathcal{L}
$$

where $\mathrm{P}^{*}\left(w / T_{l}\right)$ is the probability that $w$ wavelengths, $0 \leq w \leq W_{l} \leq T_{l}$, are in use in the link $l$, given that the link has unlimited capacity (i.e. $T_{l}$ wavelengths). The error introduced by this approximation is negligible, as shown in [23].

Because all connections have the same traffic load, $P^{*}\left(w / T_{l}\right)$ follows a Binomial distribution with parameters $T_{l}$ and $\rho$. That is:

$$
P^{*}\left(w / T_{l}\right)=\left(\begin{array}{c}
T_{l} \\
w
\end{array}\right) \rho^{w}(1-\rho)^{T_{l}-w} ; 0 \leq w \leq W_{l}, \forall l \in \mathcal{L}
$$

\subsection{Fast link dimensioning}

Let $B_{l \text { TARGET }}$ be the value of $B_{l}$ obtained from Eq. (3). Thus, each network link is dimensioned according to the following iterative procedure:

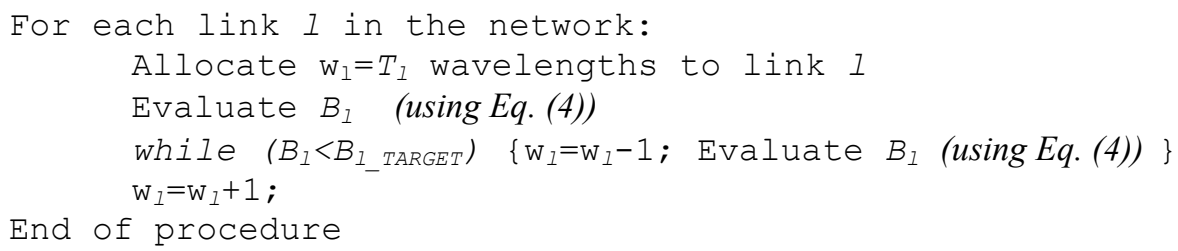

We denote this method as Traffic-dependent Link-Based (TLB) dimensioning algorithm. 


\section{Numerical Results}

The proposed dimensioning algorithm was applied to different network topologies for values of the target blocking $\mathrm{B}$ equal to $10^{-3}$ and $10^{-6}$. For comparison purposes, the following two additional dimensioning algorithms were also included:

Static Link-Based (SLB) dimensioning. Each link $l$ is allocated as many wavelengths as connections using link $l$ (the number of connections using link $l$ is determined by the fixed routing algorithm) [11-12]. This algorithm requires the minimum number of wavelengths to achieve zero blocking probability (in a wavelength-convertible network) and thus, it is used as a reference in this paper.

Homogeneous dimensioning (HD). All the network links have the same number of wavelengths. Initially, this number of wavelengths corresponds to the maximum number of wavelengths required by any link in the network when applying the static dimensioning. Then, the blocking probability is evaluated (typically, by means of simulation) and the original number of wavelengths is decreased by one until at least one of the connections achieves a blocking probability higher than the target. This type of dimensioning algorithm is commonly found in the literature; see for example [8-10].

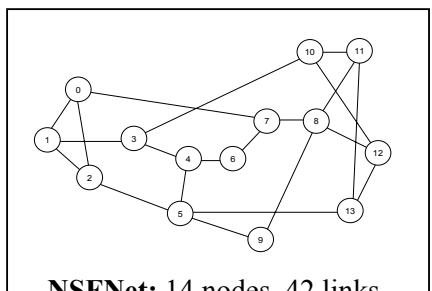

NSFNet: 14 nodes, 42 links

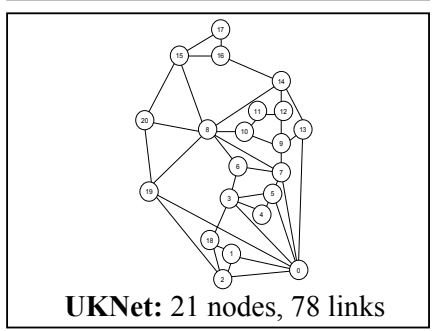

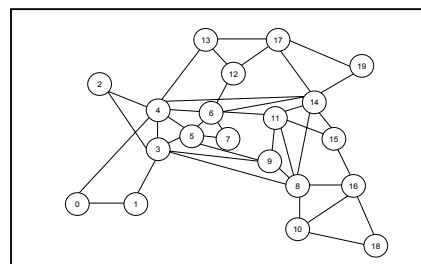

EON: 20 nodes, 78 links

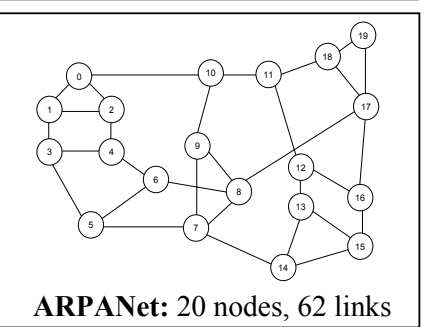

Fig. 2. Network topologies

As way of illustration, figures 3a)-d) show the results for the total network cost (that is, $\sum_{\forall l} W_{l}$, the sum of all the wavelengths required in the network links) achieved by the different dimensioning algorithms applied to the NSFNet and EON topologies with a target blocking $\mathrm{B}=10^{-3}$ and to the UKNet and ARPANet topologies for a target blocking $\mathrm{B}=10^{-6}$. 

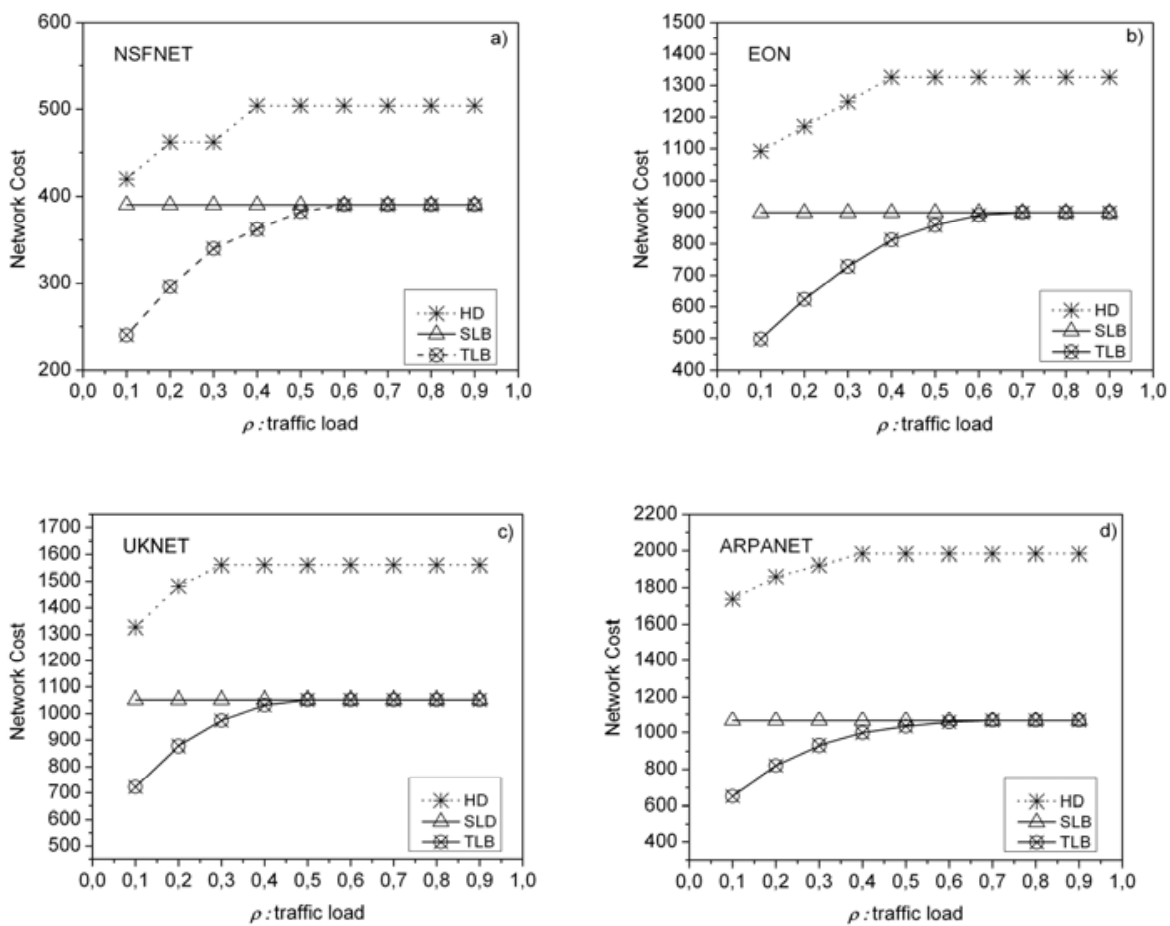

Fig. 3. Cost of a) NSFNet $B=10^{-3}$, b) EON $B=10^{-3}$, c) UKNet $B=10^{-6}$ and d) ARPANet $B=10^{-6}$ networks for the different dimensioning algorithms as a function of the traffic load

It can be seen that the TLB (Traffic-dependent Link-Based) dimensioning algorithm achieved lower or equal network cost than the SLB dimensioning algorithm. For example, for $\mathrm{B}=10^{-3}$ the cost of TLB for traffic loads under 0.6 was lower than the reference SLB algorithm for all the studies topologies, especially at low loads $(<0.3)$ where the proposed method required up to the half of wavelengths than the SLB algorithm. For traffic loads higher than 0.6 both algorithms (TLB and SLB) yield the same network cost. For values of $\mathrm{B}=10^{-6}$, the proposed algorithm performs better than the reference algorithm for loads lower than 0.4-0.5 in all the studies topologies. As most networks currently operate at low loads (typically<0.3 [24,25]), TLB will ensure the greatest benefits where it is needed most - that is in allowing the network cost to decrease whilst satisfying the required blocking.

From Fig. 3 it can also be seen that the commonly used approach of allocating the same number of wavelengths to all the links (HD) leads to the highest network cost for a wide range of traffic loads. For example, for high loads, the HD algorithm required up to 1.8 times more wavelengths than the reference SLB algorithm. Given that the reference algorithm guarantees zero blocking probability, it is highly inefficient to utilize the HD algorithm whose normalized cost is higher than 1. 
The number of wavelengths per link obtained by dimensioning the different networks of Fig. 2, with algorithms TLB and SLB was validated by means of simulation: the target blocking probability per connection was set to $10^{-3}$ and each link of the networks shown in Fig. 2 was equipped first with the number of wavelengths determined by the TLB dimensioning algorithm and then, equipped with the number of wavelengths defined by the SLB algorithm. The blocking probability of each of the $N(N-1)$ connections was evaluated for both algorithms in the different topologies (results not shown here due to space constraints). In all the cases the dimensioning algorithms proved to be effective since the maximum blocking of all connections was always slightly lower than the target. An additional advantage of TLB was its low execution time - less than one second - making it several orders of magnitude faster than the simulation-based approach of the HD algorithm.

\section{Summary}

In this paper a new algorithm, called TLB, for the dimensioning of end-to-end optical burst switched networks under ON-OFF traffic model was proposed and compared to other 2 commonly used approaches. The proposed method was proved to be effective (the target blocking was met) and better than existing approaches in allowing savings of up to half the number of wavelengths required by the static dimensioning. By being effective and fast, the proposed method is very attractive for implementation as a dimensioning tool for future dynamic OBS networks.

Acknowledgements. Financial support from Fondecyt Projects 1000055 and 1050361 (Chilean Government), USM Projects 23.07.27 and 23.07.28 (Universidad Santa María) and DIPUV project 45/2006 (Universidad de Valparaíso) is gratefully acknowledged.

\section{References}

1. Blumenthal, D. J.: Optical packet switching. In Proc. IEEE Leos Annual Meeting Conference, paper ThU1, Puerto Rico, November 2004

2. El-Bawab, T.S., Shin, J-D.: Optical packet switching in core networks: between vision and reality. IEEE Communications Magazine, Vol.40 (9) (2002) 60-65

3. Qiao, C., Yoo, M.: Optical Burst Switching (OBS) - a new paradigm for an optical internet. Journal of High Speed Networks, Vol.8 (1999) 69-84

4. Turner, J.: Terabit burst switching. Journal of High Speed Networks, Vol.8 (1999) 3-16

5. Düser, M., Bayvel, P.: Analysis of a dynamically wavelength-routed optical burst switched network architecture. J. of Lightwave Technology, Vol.4 (2002) 574-585

6. Arakawa, S., Miyamoto, K., Murata, M., Miyahara, H.: Delay analyses of wavelength reservation methods for high-speed burst transfer in photonic networks. In Proc. International Technical Conference on Circuits and Systems, Computers and Comms. (ITCCSCC99) July 1999

7. Zapata, A., de Miguel, I., Dueser, M., Spencer, J., Bayvel, P., Breuer, D., Hanik, N., Gladish, A.: Next Generation 100-Gigabit Metro Ethernet (100GbME) using multiwavelength optical rings. IEEE/OSA Journal of Lightwave Technology, special issue on Metro \& Access Networks, Vol. 22 (11), (2004), 2420-2434 
8. Teng, J.; Rouskas, G.N.: Routing path optimization in optical burst switched networks. Optical Networks Design and Modeling Conference (ONDM), Milan, February 2005

9. Yu, J.; Yamashita, I.; Seikai, S.; Kitayama, K.: Upgrade design of survivable wavelengthrouted networks for increase of traffic loads. Optical Networks Design and Modeling Conference (ONDM), Milan, February 2005

10. Kozlovski, E.; Düser, M.; de Miguel, I.; Bayvel, P.: Analysis of burst scheduling for dynamic wavelength assignment in optical burst switched networks. 14th Annual Meeting of the IEEE Lasers \& Electro-Optics Society, LEOS 2001. San Diego, California. 11-15 November 2001. paper TuD2

11. Zapata A., Bayvel P.: Dynamic Wavelength-Routed Optical Burst Switched Networks: Scalability Analysis and Comparison with Static Wavelength-Routed Optical Networks. In Proceedings of Optical Fiber Communications Conference 2003 (OFC2003), 23-28 March 2003, Atlanta, USA.

12. Gauger C. M., Kohn M., Zhang J., Mukherjee B.: Network Performance of optical burst/packet switching: the impact of dimensioning, routing and contention resolution. In Proceedings of ITG-Fachtgung Photonic Networks, May 2005, Leipzig, Germany

13. Choi, J.Y., Choi, J.S., Kang M.: Link dimensioning in burst-switching based optical networks: In Proceedings of Optical Fiber Communications Conference, OFC 2006

14. Choi, J. Y. , Vu, H.L., Cameron, C. W., Zukerman, M., Kang, M.: The effect of burst assembly on performance of optical burst switched networks. Lecture Notes on Computer Science, Vol.3090 (2004) 729-739

15. Tancevski, L., Bononi, A., Rusch, L. A.: Output power and SNR swings in cascades of EDFAs for circuit- and packet-switched optical networks. Journal of Lightwave Technology, Vol.17 (5) (1999) 733-742

16. Zukerman, M., Wong, E., Rosberg, Z., Lee, G., Lu, H.V.: On teletraffic applications to OBS. IEEE Communications Letters, Vol.8 (2) (2004) 731-733

17. Elmirghani J., Moutfah H. T.: All-optical wavelength conversion: technologies and applications in DWDM networks. IEEE Communications Magazine, vol. 38, No. 3, pp. 8692, March 2000

18. Zapata A., Bayvel P.: Optimality of resource allocation algorithms in dynamic WDM networks. In Proceedings of $10^{\text {th }}$ European Conference on Network and Optical Communications, NOC 2005, London, UK, 5-7 July 2005, pp.131-138

19. Zang H., Jue J., Mukherjee B.: Review of routing and wavelength assignment approaches for wavelength-routed optical WDM networks. Optical Networks Magazine, vol.1, pp.4760, January 2000

20. Adas A: Traffic models in broadband networks, IEEE Communications Magazine, vol. 35, no. 7, (July 1997), pp. 82-89

21. Ross S.: Introduction to probability models. $6^{\text {th }}$ Edition Academic Press, 1997, (Eq. 7.15)

22. Schwartz M.: Telecommunication networks. Protocols, modelling and analysis. Addison Wesley, 1987.

23. Vallejos R., Zapata A., Aravena M.: Fast Blocking Probability Evaluation of End-to-End Optical Burst Switching Networks with non-uniform ON-OFF Input Traffic Model. Photonic Network Communications (D.O.I. 10.1007/s11107-006-0037-y)

24. Odlyzko, A.: Data networks are lightly utilized, and will stay that way. Review of Network Economics, Vol. 2, (2003), 210-237

25. Bhattacharyya, S.; Diot, C.; Jetcheva, J.; Taft, N.: POP-Level and access-link-level traffic dynamics in a Tier-1 POP. ACM SIGCOMM Internet Measurement Workshop, Vol. 1, 3953, San Francisco, CA, November 2001 